\title{
LARGE-SCALE SOLAR MAGNETIC FIELDS
}

\author{
V. BUMBA \\ Astronomical Institute of the Czechoslovak Academy of Sciences, \\ Observatory Ondrejov, Czechoslovakia
}

\begin{abstract}
The characteristics of the large-scale distribution of the solar magnetic fields on the basis of a series of solar magnetic synoptic charts covering more than 15 years of observations are given. The major part of our information concerns the morphology and only some results deal with the kinematics of the field distribution. Results of averaged solar magnetic field fluxes and polarity reversal studies as well as of preliminary investigation of the very-low angular resolution magnetic measurements are given. The regular zonal and sectoral distribution of photospheric background fields, the different role or visibilility of structures in both polarities is discussed. The reflection of both main types of the longitudinal distribution of large-scale solar background magnetic fields (the 27-day, the 28-29-day successions, the 'supergiant' structures) in the interplanetary magnetic field distribution is also considered.
\end{abstract}

\section{Introduction}

Existence of the solar magnetic fields outside sunspots has been demonstrated and discussed for the first time by H. W. and H. D. Babcock (1955). They showed the reality of a polar field and two types of the fields at lower heliographic latitudes: Bipolar Magnetic Regions (BMR's) and Unipolar Magnetic Regions (UMR's). Thanks to synoptic charts drawn from the daily magnetograms of the whole solar disk obtained regularly since August 1959 (Howard et al., 1967) at the Mt. Wilson Observatory with a new magnetograph we have learned some more characteristics of the solar background magnetic fields distribution. The technique of the construction of the synoptic maps which fix the magnetic field situation around its central meridian passage once per solar rotation makes them suitable sources of our knowledge of the large-scale distribution of solar magnetic fields having appropriate long life-times.

One of the most important facts we have to pay attention to during the study of these large-scale fields is the angular resolution of the observational instrument. For example, the older Babcock's magnetograms made during 1953-1954 with an angular resolution of about $38^{\prime \prime} \times 70^{\prime \prime}$, although they have not yet been fully evaluated, seem to demonstrate - at least in several up to now utilized cases - a different conception of the weak background magnetic field distribution from what we used to receive on the daily maps or synoptic charts published in the Mt. Wilson Atlas of Solar Magnetic Fields (Howard et al., 1967) having the increased 23" $\times 23^{\prime \prime}$ resolution or even on today's magnetograms and charts obtained with the still increased angular resolution of about $12^{\prime \prime} \times 10^{\prime \prime}$. With the growing resolution the large unipolar areas of BMR's or UMR's disintegrate into smaller mixed polarity islands and we are getting more and more confused by the complexity of the figure.

During the last decade a rich information concerning the distribution of the solar large-scale magnetic fields has been obtained from direct measurements, as well as from the distribution of the interplanetary magnetic fields reflecting the organization of the solar large-scale fields in a surprising manner, and from the investigation of various activity phenomena spacing on the solar surface. 
In this paper we would like to summarize the most characteristic features of the large-scale solar magnetic fields as they have been found during the recent few years. The large-scale in our case means that the dimensions of the investigated fields are greater than the scale of one active region.

\section{Average Solar Magnetic Field Fluxes and Polarity Reversal}

To understand better the rules following which the large-scale fields are distributed we may start with the problem of the Sun seen as a star. Howard (1974a, b, c) just recently examined the magnetic flux data, average magnetic field strengths and changes of the field polarity from the Mount Wilson magnetographic data over the time interval 1967-1973. He found that the fact that on the whole the activity in the northern solar hemisphere was greater than that in the south during the mentioned interval, is reflected in the $7 \%$ difference in total flux values between the two hemispheres in favour of the northern one. This $\mathrm{N}-\mathrm{S}$ percentage difference is greater at the highest heliographic latitudes, where above $70^{\circ}$ there is about one third more flux in the north than in the south. But in regard to the total amount of the solar magnetic flux, it seems, that about $95 \%$ of it is connected with latitudes below $40^{\circ}$ in both hemispheres and that the flux above heliographic latitude $60^{\circ}$ represents less than $2 \%$ of the total solar magnetic flux.

Activity-cycle-related increase in the total magnetic flux may be seen on both hemispheres and it is strongest in the activity zones but it may be seen at the higher latitudes as well. The negative flux is stronger than the positive magnetic flux at all latitudes around the time of solar maximum. This effect is proportionally strongest poleward of $40^{\circ}$ where a 'wave' of the negative flux may be seen moving poleward.

It is known to observers and Howard (1974a, b) has demonstrated it again, that at times the fields appear, their polarity behaves in the same way over the entire solar disk or at least in a large portion of it. He brings two nice examples of it - the large positive 'spike' in the middle of 1972 caused by only a 'handful of days' observations with strong positive bias and the negative surge early in 1967. It is interesting that these biases in polarity of the solar magnetic field are reflected in biases of the same polarity in the interplanetary magnetic field (Wilcox, 1972). Thus the 'monopole' behaviour of the solar magnetic fields observed since the earliest magnetographic observations (H. W. and H. D. Babcock, 1955) seems to be a real effect as yet without explanation.

As in the polar field polarity reversal during the maximum activity of the previous solar cycle (Babcock, 1959), during the present cycle the south polar fields changed sign significantly earlier than did the north polar fields. But that reversal was a weaker one. The polar fields $\left(\varphi>70^{\circ}\right)$ reversed in about September, 1969, and the $40-50^{\circ}$ zone reversed in March, 1968 . The most striking polarity reversal, that in the north, occurred approximately two years after the activity maximum. The north polar fields changed sign from weakly negative to rather strongly positive in August, 1971. This high-latitude reversal was accompanied by a rather general positive surge in field strengths over the whole solar disk. The fields at successively lower latitudes in the north changed sign earlier, as if there were a positive 'wave' starting in the 
$40-50^{\circ}$ zone and reaching the pole in about one year. No evidence has been found for a related reversal in the equatorial zone. On the other hand Wilcox and Scherrer (1972) have shown from interplanetary field data inferred by Svalgaard (1972) from polar geomagnetic data that a change in phase of the predominant polarity of the interplanetary field occurs on the average $2.75 \mathrm{yr}$ after activity maximum.

The average fields poleward of $40^{\circ}$ in each hemisphere have over the whole period investigated by Howard (1974a) the sign of the follower spots, and the fields equatorward of $40^{\circ}$ have the sign of the leader spots.

\section{Large-Scale Distribution of Magnetic Fields on the Solar Disk Obtained with Very LowAngular Resolution}

As a next step to make a transition from the magnetically wholly integrated Sun to its solar field distribution I would like to present as an example a few daily magnetic maps constructed from the Babcocks' low resolution $\left(38^{\prime \prime} \times 70^{\prime \prime}\right)$ magnetograms obtained during the period of minimum activity in 1953. One of the advantages of those magnetograms seems to me the fact that they make it possible to estimate the boundary between the opposite polarities. Thus they visualized with a high degree of integration the distribution of very weak, highly integrated background magnetic fields.

The pictures available up to now display the three typical situations of the field distribution we may observe on such maps:

(a) Practically each solar hemisphere is occupied by one polarity field (the northern hemisphere by the positive polarity field, the southern hemisphere by the negative one) (Figure 1a).

(b) The leading magnetic fields of active regions are formed as gulfs of an opposite polarity reaching the given hemisphere from the opposite hemisphere. In other words, during 1953 the active regions developed in the northern hemisphere from the declining 18th cycle of activity have the polarity of their following portions the same as the background magnetic field of this hemisphere, that is the positive polarity. The leading parts of these active regions have the negative polarity and are formed from gulfs of this polarity reaching the place at the northern hemisphere from the other side of the equator as a prolongation of the southern negative polarity background field. The same is true but vice versa about the active regions on the southern hemisphere (Figure 1b, c).

(c) In this still more complicated situation the northern polar fields are positive, the southern polar fields are negative, but in activity zones the polarities of the background fields are reversed in such a way that the northern activity zone is negative and the southern one is positive. This is practically the same situation as mentioned above when speaking about Howard's results for 1967-1973: the Sun has four parallel zones with alternating polarities, two of them are positive and two of them are negative (Figure 1d). In heliographic longitudes with active regions the situation is, of course, much more complicated.

I do not mean to say that the mentioned three types of situations are the three evolutionary stages of the activity development. But I would like to underline the 

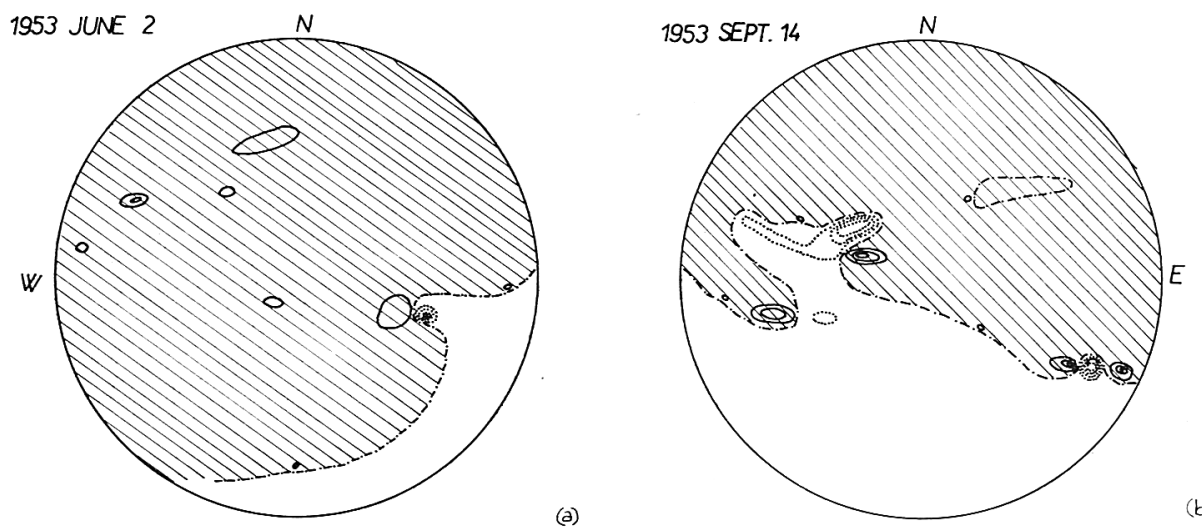

(a)

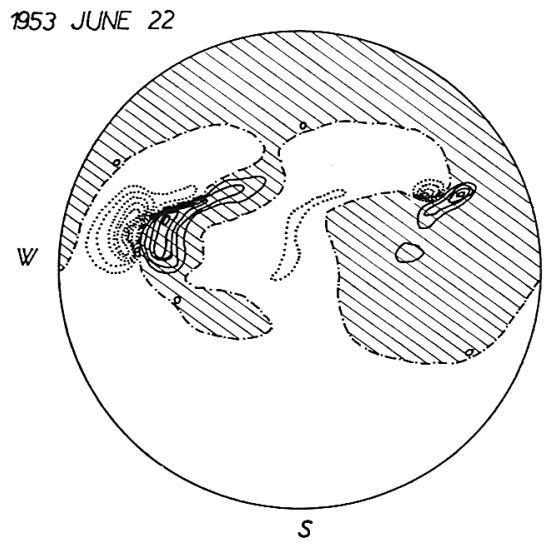

(c)

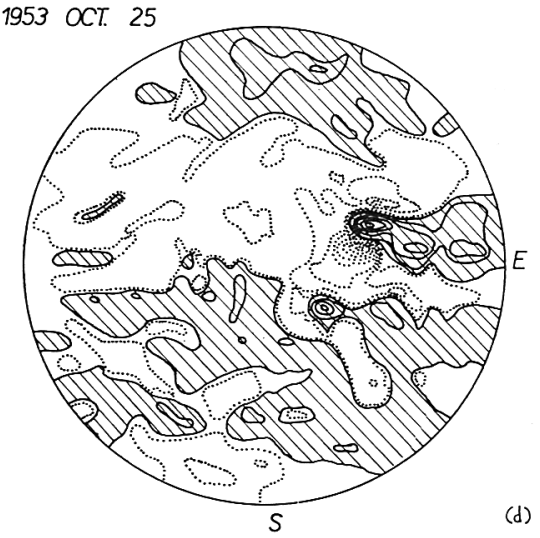

Fig. 1. Four daily magnetic maps constructed from the Babcocks' low resolution $\left(38^{\prime \prime} \times 70^{\prime \prime}\right)$ magnetograms obtained during the period of minimum activity in 1953 . The distribution of highly integrated background photospheric magnetic fields is visualized. (Negative polarity is indicated by dotted lines and shadowed areas, the positive one by full lines and parallel hatching.)

(a) 1953, June 2; one of the most simple situations.

(b) 1953, Sept. 14; one gulf of leading polarity in the northern hemisphere has been formed from the negative polarity main field of the southern hemisphere.

(c) 1953 , June 22;

(d) 1953, October 25; the Sun has four parallel zones with alternating polarities, two of them are positive and two of them are negative.

interesting fact of the identity of the following magnetic field with that of the background field of the given hemisphere. This may be, for example, the reason why the active regions start to develop from their following portions first (Bumba, and Howard, 1965; Knoška, 1976) etc. A lot of work is needed to investigate further this question. But I believe that more observational material with very high degree of integration, i.e. with low angular resolution, is desirable. 


\section{Longitudinal Distribution of Solar Magnetic Fields}

More than five years ago (Bumba and Howard, 1969) certain new patterns in the large-scale field distribution were characterized: Sections are the features of one polarity developing in activity zones from the magnetic fields of one to several active regions. They extend generally across the equator and they may be followed often for at least 10 rotations. Series of sections of one polarity on consecutive synoptic charts mounted in chronological order form rows. Two or more rows of one or both polarities may constitute a nearly continuous stream.

It has also been shown that the sections in the equatorial zone $\left(\varphi= \pm 20^{\circ}\right)$ have their recurrence period near to 27 days which means that they are slightly shifted to the west from one rotation to the following one, if drawn in Carrington's coordinate network, and the rows they form are then slightly inclined. This inclination practically disappears if drawn in Barthel's coordinates. Rows from opposite polarities differ at the given moment by various widths and level of concentration of their fields, sometimes also by their inclination. Sometimes the rows of the positive polarity, at other times those of the negative polarity are more pronounced.

In higher heliographic latitudes $\left(\varphi> \pm 20^{\circ}\right)$ the rows drawn in Carrington's coordinates are inclined in the opposite direction if compared with the equatorial rows and their inclination angle is greater. This inclination seems to be connected with the 28-29 day synodic rotational period of the sections developed in those latitudes.

The same characteristic patterns with the same properties described above may be now found during the whole material of the synoptic charts as yet available (1959-1975) (Bumba, 1976), (Figures 2 and 3).

During the descending part of the previous cycle as well as during the whole present cycle the evolution of the rows demonstrates a typical behaviour: a row starts in the form of a narrow section. The width of the section grows from one rotation to the next until it reaches its maximum of development. This maximal phase is usually well defined and visible in between the other background fields, due to the high degree of the field concentration and to the relatively large area occupied by the section. Afterwards the section of such a row narrows rather fast and disappears again in the noise of the background field. What is more important, the individual rows reach their maxima successively in such a way that with the decreasing heliographic longitude of the row the development of its maximum occurred later. The succession of consecutively developed maxima of one polarity rows forms a second type of 'row' inclination which in Carrington's coordinates goes parallel to those we may see in higher heliographic latitudes $\left(\varphi> \pm 20^{\circ}\right)$. This indicates that the synodic period of the rotation of these 'secondary rows' is also close to 28-29 days. These 'secondary equatorial rows' which are as a rule more pronounced in the negative polarity fields during both cycles of the activity for which we have the observational material develop simultaneously with the higher latitudinal zone rows (Figures 4, 2 and 3).

It may be underlined that the mentioned two basic inclinations of the rows or two basic periods of the synodic rotation of the sections in the large-scale distribution of the solar magnetic fields have been already demonstrated in several manners of their 

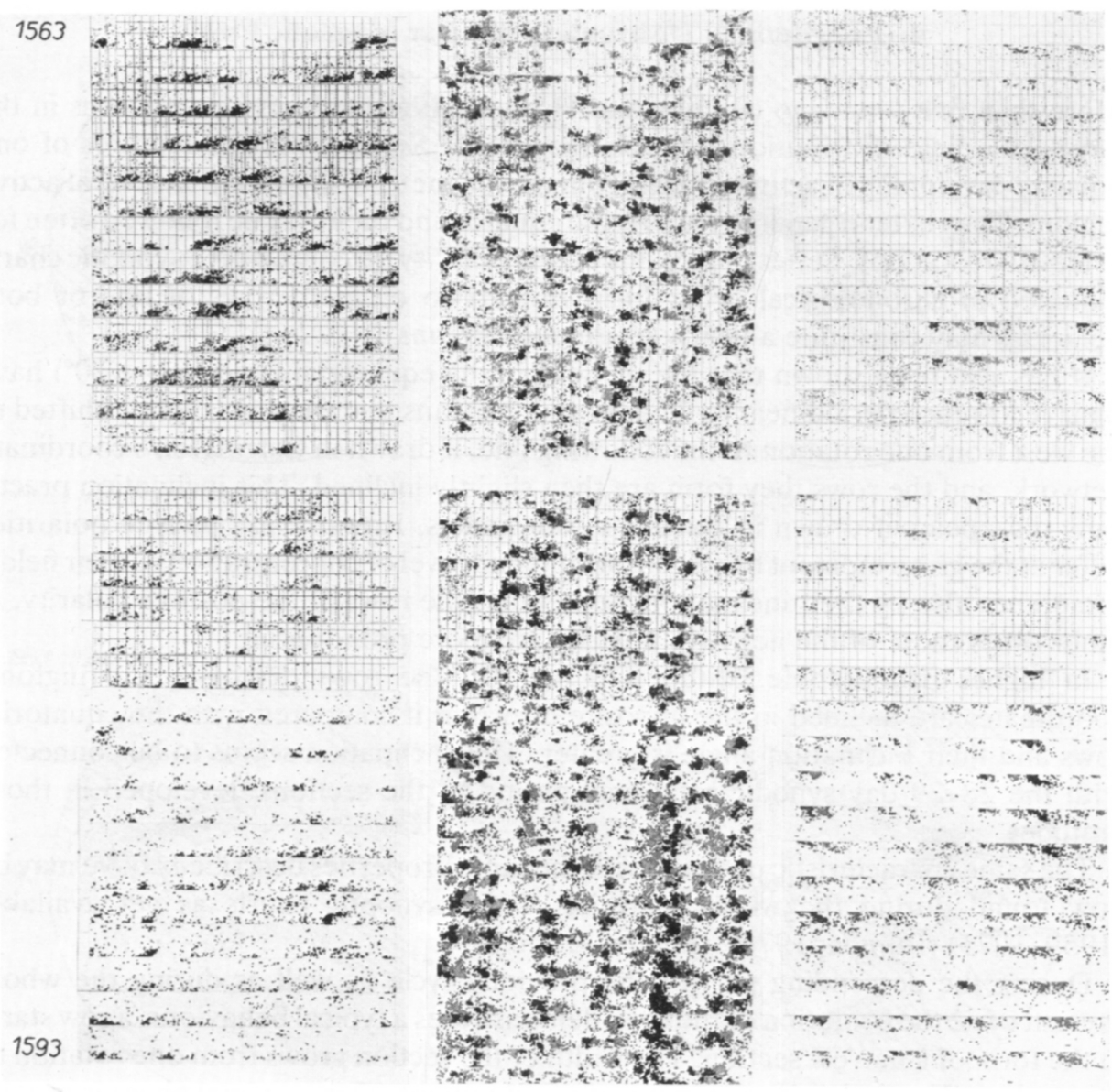

Fig. 2. Magnetic synoptic charts cut into strips representing two zones of latitude $\left( \pm 20^{\circ}, \pm 20^{\circ}- \pm 40^{\circ}\right)$ and mounted in chronological order during the maximum phase of the present cycle of solar activity (Rotations Nos. 1563-1593). The middle line represents the consecutive equatorial strips of the individual synoptic charts, the adjoining data represents the strips of higher latitudes on the Northern (left) and Southern (right) hemispheres. The dark regions represent the minus polarity areas.

manifestation (Ambrož et al., 1971; Bumba, 1970; Bumba et al., 1968; etc.). It seems even that these two main types of the rows (or streams) determine in the mutual interaction the morphology and may be also the dynamics of the background magnetic field distribution: the most interesting structures develop in the place and time when these two successions intersect.

Just recently Svalgaard and Wilcox (1974) investigated the same large-scale structures of the solar magnetic field using the polarity of the interplanetary magnetic field as inferred from polar geomagnetic observations. They succeeded to study the material for the past five sunspot cycles. I believe their results are very often in a good agreement with those obtained from the direct research of the solar magnetic fields. But since Dr Wilcox will tell us the main topics of their study in a contributed paper, I will not touch this question any more. 

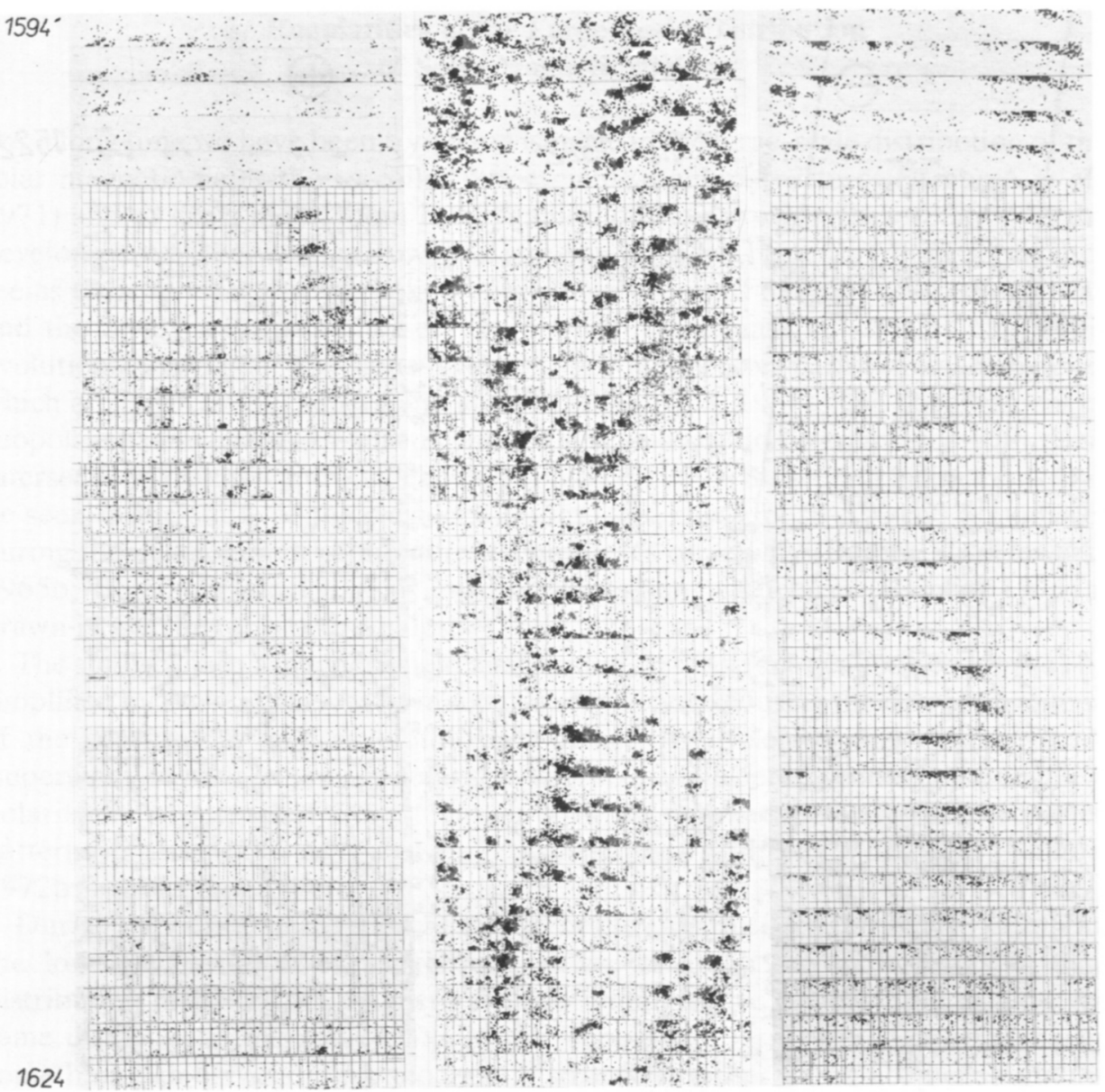

Fig. 3. Magnetic synoptic charts of solar photospheric fields organized as in Figure 2 during the descending part of the present cycle of solar activity (Rotations Nos. 1594-1624).

During the recent cycle of the activity two in longitude practically opposite complex magnetic streams used to exist on the solar surface, in each of which the opposite polarity seemed to be predominant. During the present activity cycle this regularity in distribution seems to lose its evident character one or two years after the maximum of the solar cycle.

Longitudinal distribution of the large-scale solar magnetic fields has also been many times studied in connection with the problem of the so-called 'Active Longitudes' (Vitinskij, 1969; 1973). Just recently Vitinskij (1974) using also the Mt. Wilson Observatory synoptic maps as initial data and applying his methods of isoline charts found several such active longitudes or concentrations of photospheric background magnetic fields but only one of these active longitudes is retained for each field polarity during the 19th and 20th cycle of activity. The other active longitudes either disappeared or are shifted in longitude. The conclusion made from 


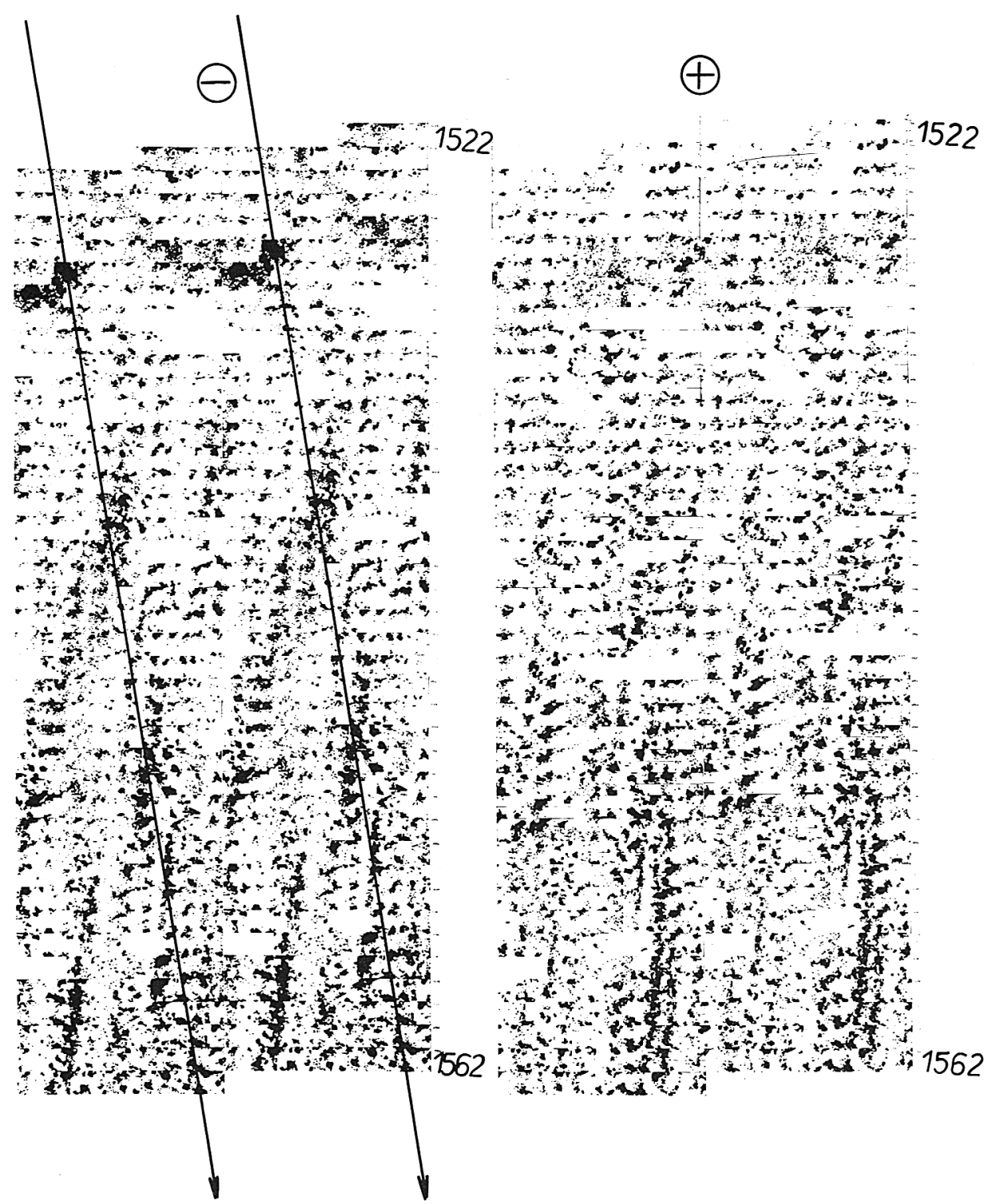

Fig. 4. Magnetic synoptic charts cut into equatorial strips $\left( \pm 20^{\circ}\right)$ and mounted in chronological order (each strip of synoptic chart is drawn twice) for Rotations Nos. 1522-1562. Minus polarity is on the left, plus on the right of the figure. The main inclination of 27 days can be seen as well as on the minus polarity drawings the intensification of subsequent individual magnetic rows, starting in the upper left-hand corner and going in a succession forming the 28-29 day secondary row.

these observations by the author is as follows: the latitudinal-longitudinal distribution of photospheric background magnetic fields is not very stable in time (the time-scale here is one activity cycle). Therefore, we cannot be sure that they are the basis of active longitudes found from other indices of activity. 


\section{Regularities in the Large-Scale Distribution of Solar Magnetic Fields}

For a long time we have been trying to visualize in the large-scale distribution of the solar magnetic fields the so-called 'supergiant' regular structures (Ambrož et al., 1971) and to learn more about the dynamics of their growth, their relation to the development of the sections, rows and streams. As it has been said, their occurrence seems to be related to those heliographic longitudes and times in which the 27-day and the 28-29 day rows intersect or in which the equatorial streams have their evolutionary maxima. The same has been shown in proton-flare activity complexes which are parts of the 'supergiant' structures (Švestka, 1968). The development of subpolar maxima of green coronal emission seems also to be connected with these intersections (Bumba and Růžičková-Topolová, 1969). Both main inclinations may be seen in the shifts of the western (27-day) and eastern (28-29-day) boundaries during the development of the normal complexes of activity (Bumba and Howard, 1965b; Bumba et al., 1968) or even of 'supergiant' structures (Bumba, 1976) if drawn in Carrington's coordinates.

The study of such a magnetic field distribution on the whole synoptic charts may be simplified by drawing the charts in separated polarities. During the decreasing phase of the previous as well as of the present activity cycle we may observe these 'supergiant' regular or semi-regular structures - usually better visible in the negative polarity fields - as they form together with the positive polarity fields complex patterns in which both polarities are complementary (Ambrož et al., 1971; Bumba, 1972b; Bumba and Sýkora, 1973).

During the period of the minimum activity they are not seen, probably because of the low concentration of background fields, although the main knots in field distribution formed from magnetic fields of young active regions used to have the same distances as the main knots of the 'supergiant' structures. Sometimes even something like halves of the 'supergiant' structures either in the northern or in the southern hemisphere are observable. During the maximum of the present cycle of activity the huge regular structures seem to start to be apparent again. But probably because of a still relatively low background field concentration, before all in the equatorial zone, their portions in this zone are recognizable with difficulty. It seems also that during this phase of activity cycle their life-time is shorter if compared with that during the decreasing part of the recent cycle when the greater magnetic field concentration due to the higher activity level may be the reason why they disappear more slowly below the level of detection. The same is true about the visibility and duration of the 'supergiant' structures if we compare the decreasing parts of the present and recent cycle. Thus the level of background field concentration plays probably an important role in their formation. The different level of activity in both solar hemispheres during the declining phase of the present cycle influences the situation as well.

Examining the time development of large-scale field structures in relation to the role of the equator on it, relatively often we may see that the activity develops first in higher latitudes of one hemisphere, usually the northern one, than its center of gravity, and the resulting magnetic fields shift slowly - if followed in successive 
rotations - to the lower latitudes, cross the equator and continue to shift to the higher latitudes of the opposite hemisphere and vice versa. Such kind of 'latitudinal activity oscillations' may be sometimes seen repeated several times.

We have still to say a few words about the morphology and dynamics of development of the 'supergiant' structures. They are drop-shaped with the tail in higher latitudes. The drop with its head to the west, formed from older activity regions, more stable and regular, is usually about $90^{\circ}-100^{\circ}$ long. The center of gravity of the major solar activity is located at the eastern part of the feature, where the activity changes more rapidly, just below the root of the tail. The area of positive polarity forms a 'mirror' image to that of the negative polarity with its tail stretched out to the higher latitudes of the opposite hemisphere and with its body fitting into the drop of the negative polarity feature (Figure 5). The development of the described magnetic patterns is a very complex process, because morphologically the structures of both magnetic polarities do not develop simultaneously and in phase with regard to time and heliographic longitude. The life-time of such structures is of the order of one year although they can be best observed only for a few rotations (Figure 6).

The main drop-shaped body has also its own internal structure in which we may recognize the 'giant' - as we believe granular-like - structures during the periods of best visibility. They have a diameter of the order of $30^{\circ}-35^{\circ}$ and form clusters. Several years ago we tried to demonstrate that these 'giant' granules belong to a hierarchical system of convective motions (granulation, supergranulation and giant cells). They are better visible in the positive polarity distribution, which speaks in favour of a different depth of magnetic fields with different polarities and of a different interference of both polarities with old fields and motions during an active region formation (Bumba, 1970; 1967).

\section{Averaged Synoptic Charts and Spherical Harmonics of the Large-Scale Photospheric Field}

Although all the above discussed patterns of the large-scale distribution of the background solar magnetic fields are evident on the Mt. Wilson daily magnetic maps and especially on synoptic charts representing the whole solar surface, it is very difficult to describe the patterns in a more objective way.

Stenflo (1972) used the digitized Mt. Wilson data (Altschuler et al., 1971) covering an 11-year period (August 1959-May 1970) to average each of the four existing synoptic charts into one so that a series of averaged magnetic synoptic charts might be studied. Then he constructed a butterfly diagram of the averaged magnetic fields and a sector diagram of the photospheric magnetic field averaged either over all the heliographic latitudes or for a latitudinal interval between $\pm 8^{\circ}$ only for the whole 11-year period.

Altschuler et al. $(1971,1974)$ divided the large-scale photospheric field into spherical harmonics. This procedure provides a description of the distribution of solar fields in terms of a set of objective parameters which can be visualized in certain geometrical configurations. The paper describes the photospheric field for a period of 13 years (August 1959-January 1973). 

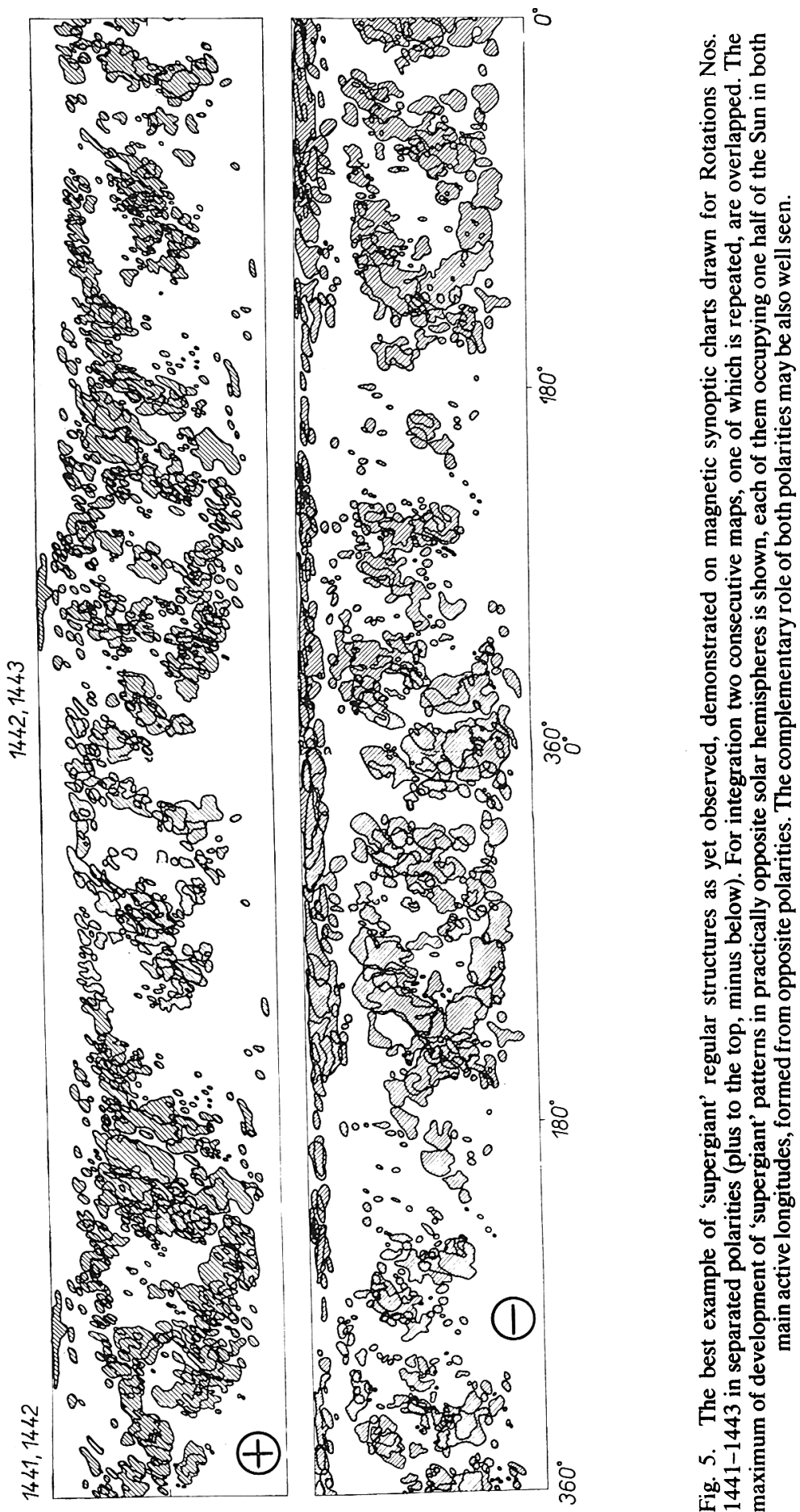

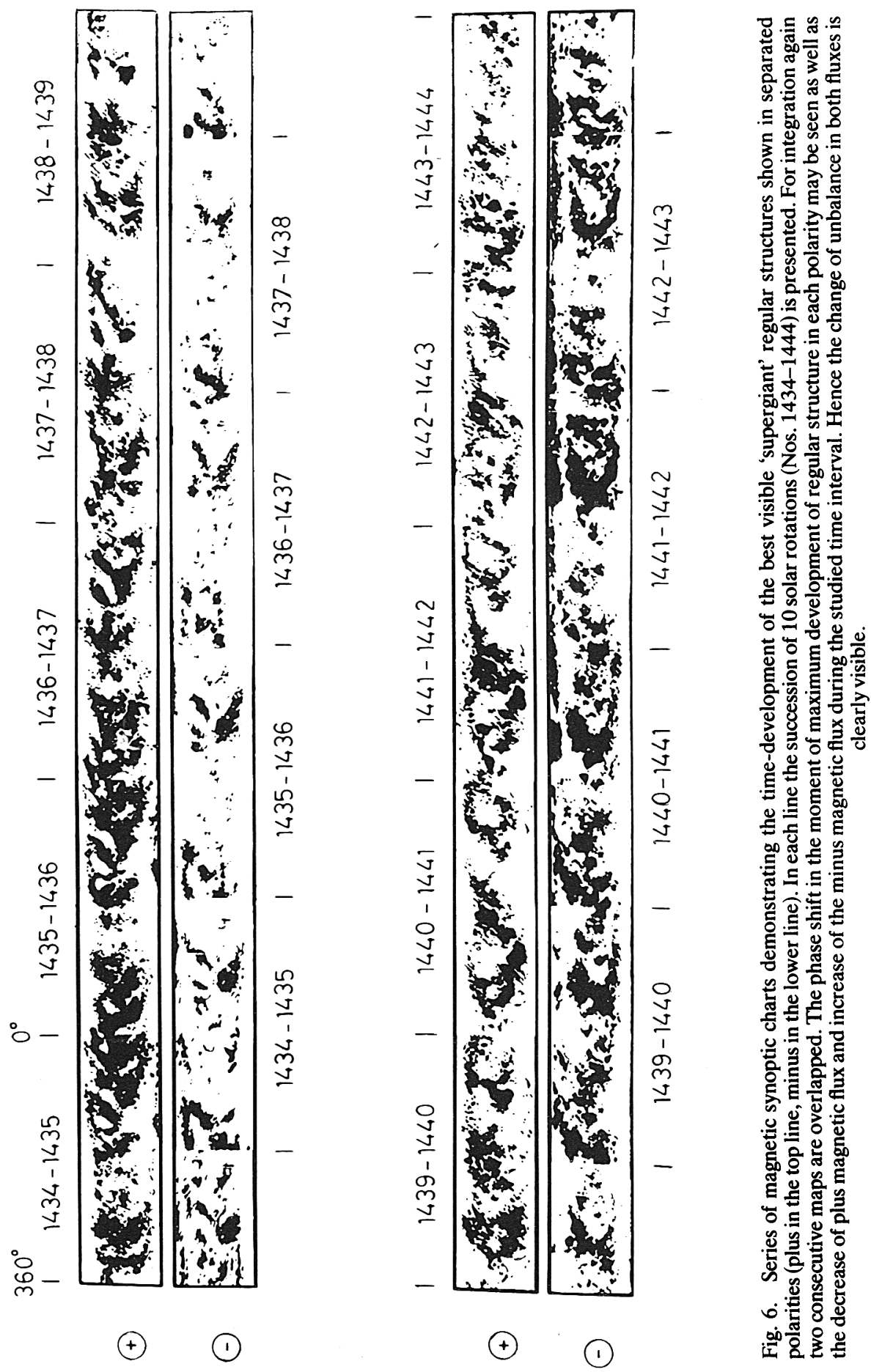
If we compare the determined dominant and important harmonics of the global photospheric field and the changes in the relative rankings of the harmonics over the time interval during which the material has been investigated by all the above mentioned methods, in other words if we compare the data represented on Figure 3 from Altschuler et al. (1974) paper with Stenflo's (1972) averaged figures or with our consecutively mounted integrated whole synoptic charts or their parts, we may see a good agreement of all the 'objective' as well as 'subjective' parameters representing the background field distribution. For example, investigating Stenflo's butterfly diagram (Figure 2; Stenflo, 1972) and the time sequence of important sector and zonal harmonics (Figure 3; Altschuler et al. 1972) for the period of the last activity minimum and for the increasing stage of the present activity cycle, we may see not only the prevailing dipole character of the solar magnetic field on both figures during the years 1963-1964 and the increasing importance of the zonal field component during the years 1965 till 1967; but comparing these data with the old Babcock's low resolution magnetic maps from the activity minimum in 1953-1954 - which we have demonstrated in the third section in the form of three typical magnetic field distributions - we may recognize in both materials again the same situation.

The same is true if we look at Stenflo's sector diagrams, especially at the one with averaged fields closer to the equator $\left( \pm 8^{\circ}\right.$; Stenflo, 1972) and if we compare it with the time variations of zonal and sector harmonics and with our diagram of longitudinal distribution of background magnetic fields from equatorial zones $\left( \pm 20^{\circ}\right)$ or with Stenflo's averaged synoptic charts. We may mention as an example the four sector character of the field in 1962, 1966 etc. Even the dominant behaviour of the $n=m=5$ harmonic preceding the development of August 1972 proton-flare region may be well seen on our figure of the equatorial rows distribution as well as in the development of a 'supergiant' structure in which the proton-flare region characteristically developed (Figure 7).

\section{Large-Scale Distribution of Solar Magnetic Fields and Solar Activity Phenomena}

It has been demonstrated several times that the distribution of solar background magnetic fields is the result of the expansion, weakening, stretching by differential rotation of magnetic fields of the old active regions and their interaction with neighbouring fields, and of the continuing development of magnetic fields of new regions within the patterns (Bumba and Howard, 1965a). Thus the dynamics of background magnetic field development is in a close relationship with the distribution of the active regions and vice versa. Consequently the same is true about the other activity phenomena linked to active regions. It has been shown, for example, that the active regions producing the particle-emitting flares occur in certain well defined places of regular 'supergiant' structures (Bumba, 1972a; Bumba and Sýkora, 1973; Bumba and Sýkora, 1974). Correlating the synoptic charts of the green ( $\lambda 5303 \AA$ ) coronal emission drawn in the form of isophotes from coronal data which were reduced to a unified photometric scale (Sýkora, 1971; 1973) with the position of 'supergiant' structures, we may see that the big elliptical features formed from an 


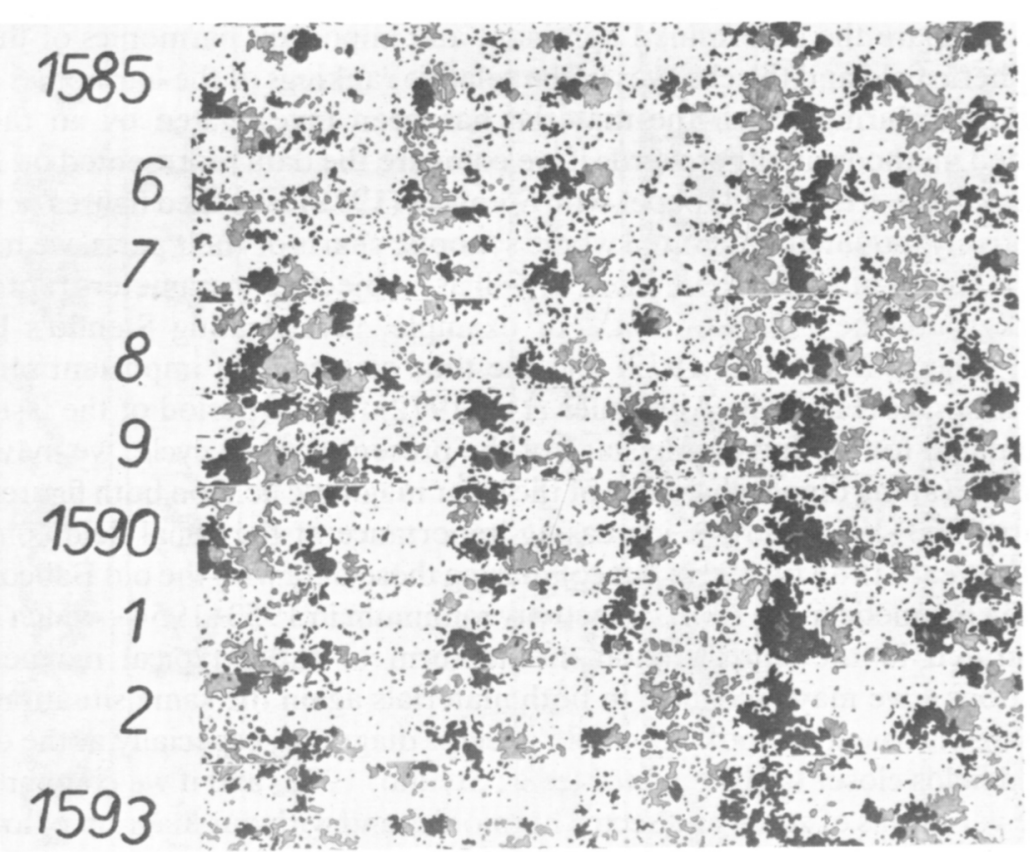

Fig. 7. Magnetic synoptic charts cut into equatorial strips $\left( \pm 20^{\circ}\right)$ and mounted in chronological order for the time period (Rotations Nos. 1585-1593) when the dominant behaviours of the $n=m=5$ harmonic preceding the development of the August 1972 proton-flares may be seen.

enhanced coronal emission practically conform with the 'supergiant' body of the magnetic field (Bumba and Sýkora, 1974).

The longitudinal distribution of the green coronal emission is closely related to the longitudinal distribution of individual polarities of the background magnetic field. Even the presence of coronal holes is demonstrated in the longitudinal distribution of the green coronal emission when its minima occur in the longitudinal intervals; they are minimally occupied by negative polarity fields. But on the other hand they agree with the position of the positive 'supergiant' body of the solar field (Bumba and Sýkora, 1973; 1974).

Using Sýkora's (1973) basic coronal data a lot of papers have appeared recently correlating the coronal structures with the distribution of interplanetary magnetic field and therefrom assessing the situation in the position of solar field sector boundary (Antonucci, 1974; Antonucci and Svalgaard, 1974). There are other papers demonstrating the situation in the corona around the magnetic field sector boundary, as, for example, by Wilcox and Svalgaard (1974) and Howard and Koomen (1974).

\section{Interplanetary Magnetic Field Sector Structure and Large-Scale Distribution of Solar Magnetic Fields}

The interplanetary magnetic field often shows a remarkably simple pattern (Wilcox and Ness, 1965) which correlates with the large-scale-averaged photospheric back- 
ground magnetic field. (Ness and Wilcox, 1966; Wilcox, 1968; Schatten et al., 1968; Wilcox and Howard, 1968). This is the reason why many authors use the satellite interplanetary magnetic field measurements as the source of information concerning the solar magnetic field distribution. But it is very difficult to visualize in the distribution of solar background fields the most apparent structure of the interplanetary field - its regular alternation of sectors with opposite polarity. The only exception is the mean photospheric magnetic field of the Sun seen as a star - where each change in polarity of the mean solar field is followed about 4.5 days later by a change in polarity of the interplanetary field (Wilcox et al., 1969; Severny et al., 1970). That is why many papers trying to correlate the position of the interplanetary sector boundary with the distribution of various activity phenomena on the Sun were published during the recent years. Not only a specific situation in the occurrence of active regions toward the position of such a sector boundary has been formed (Wilcox, 1968; Schatten et al., 1968); but the higher frequency of solar flares in connection with this boundary (Bumba and Obridko, 1969), the location of coronal condensations to the west of this boundary (Martres et al., 1970), the influence of interplanetary field sector polarity on the orientation of radio emission polarization (Krüger et al., 1968) etc. have been demonstrated. Several papers investigating the relation of coronal magnetic structures to the sector boundary showed that there is a certain difference between the boundaries changing the sector field polarity from the plus to the minus and from the minus to the plus. (Antonucci and Svalgaard, 1974). The same is true for the flares - they demonstrate a marked increase of occurrence in connection with the minus/plus sector boundary (Dittmer, 1974).

But in what relation is the position of the sector boundary to the regular 'supergiant' structures? Just recently (Bumba, 1976b) we succeeded in finding that the negative polarity interplanetary sectors coincide with the main body of the negative 'supergiant' structure (Figure 8). The minus/plus sector boundary lies close to the east end of this elliptical body which means that it precedes slightly the region of high activity occurrence. The plus/minus sector boundary separates the head of the negative field structure from the old positive polarity field. Even the longitudinal shift of the sector boundaries if examined in successive rotations goes very often parallel to that of the solar magnetic field rows and streams on equatorial $\left( \pm 20^{\circ}\right)$ strips of synoptic charts mounted successively, which means that the synodic rotation of sector boundaries is often close to 27 days. At the same time the centers of gravity of successive interplanetary field sectors (with decreasing heliographic longitude) are shifted to the east with the synodic rotation close to 28-29 days. Thus both main types of the longitudinal distribution of large-scale solar background magnetic fields (the 27-day and 28-29-day successions) are reflected in the interplanetary magnetic field distribution (Figures 9 and 10).

\section{Conclusion}

The main purpose of this talk was to show what we know up to now about the large-scale distribution of the solar magnetic fields on the basis of a series of solar magnetic synoptic charts covering more than 15 years of observations. The major 


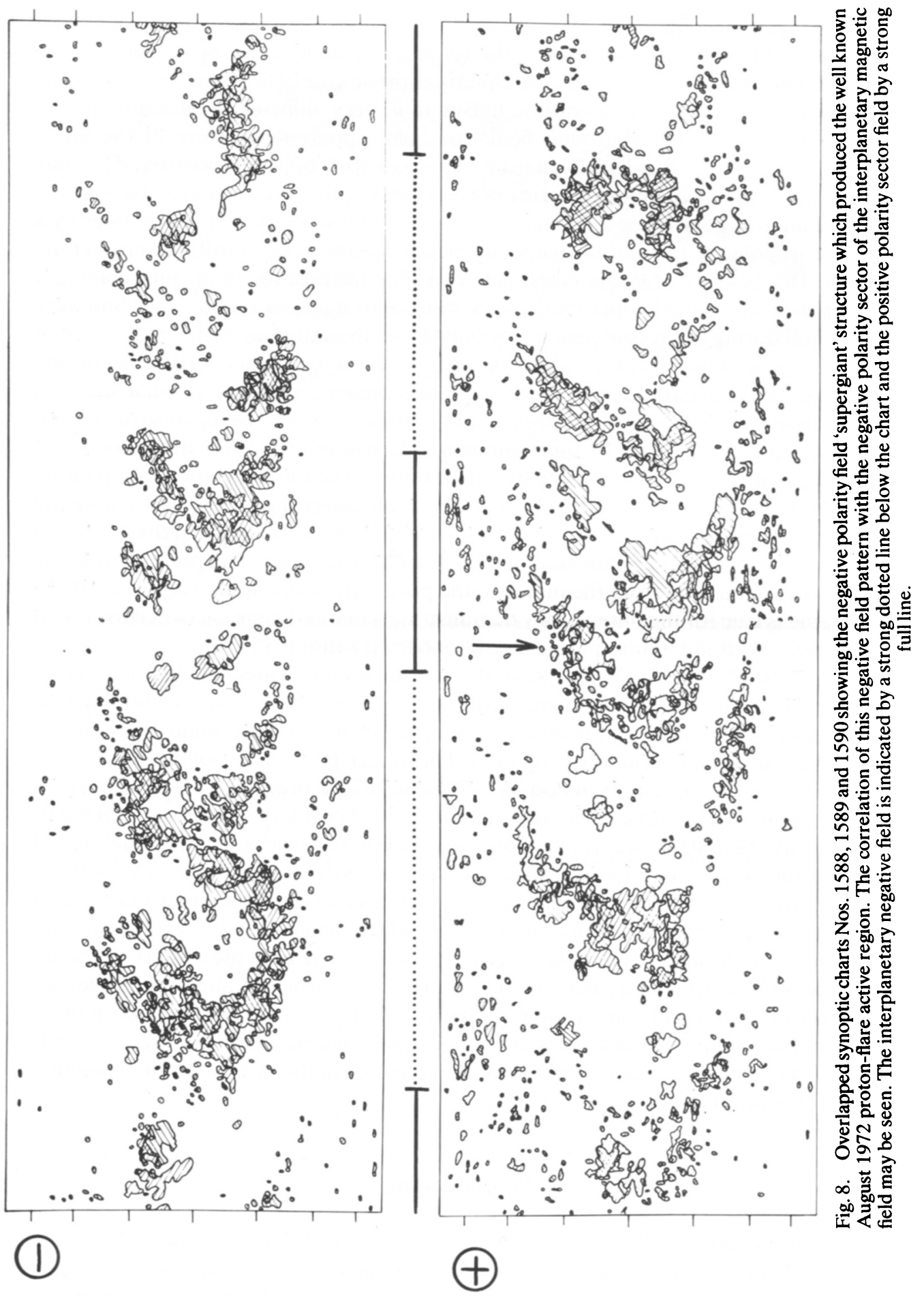




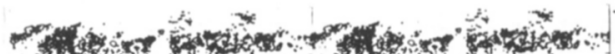

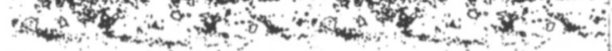

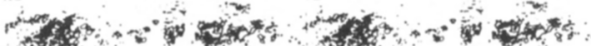

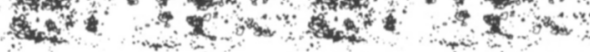

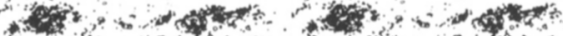

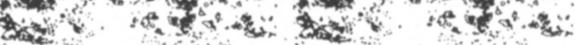

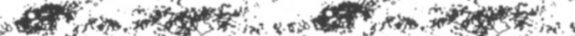

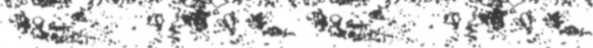

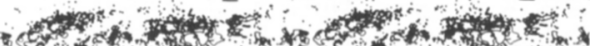

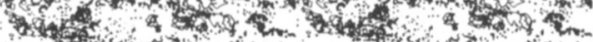

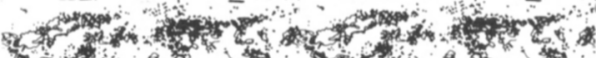

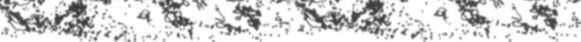

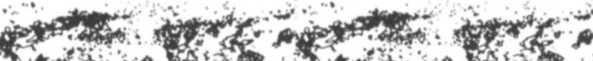

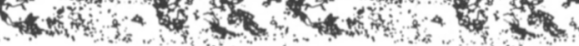
ond

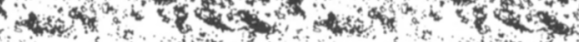

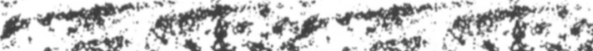

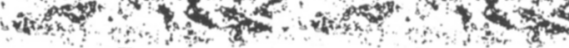

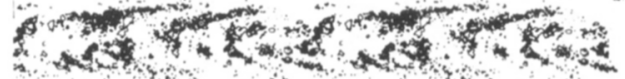

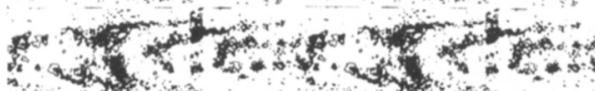

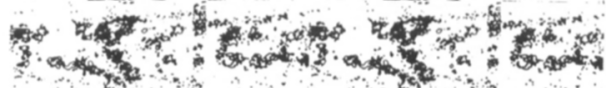

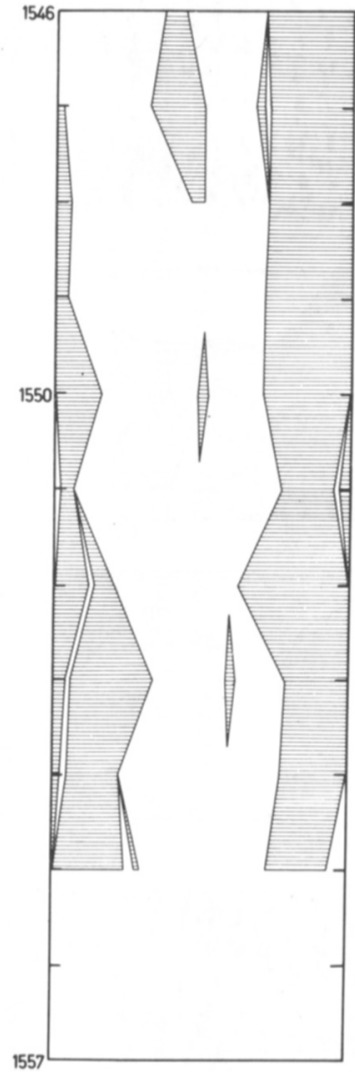

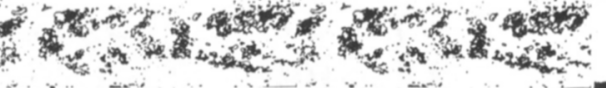

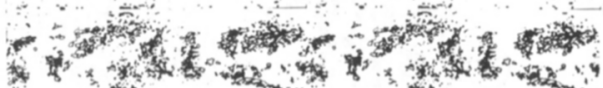

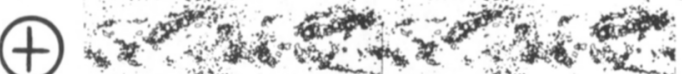

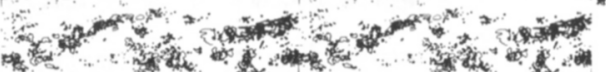

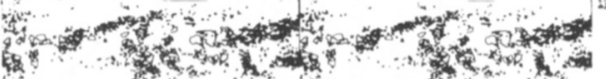

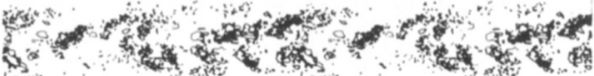

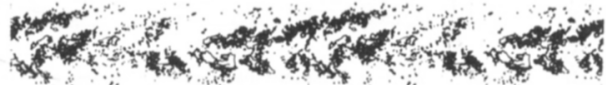

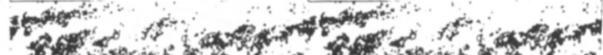

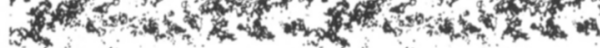

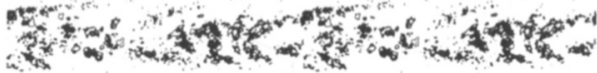

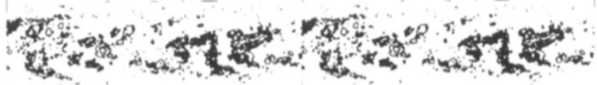

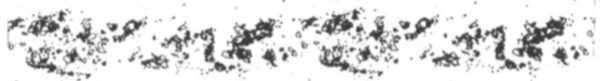

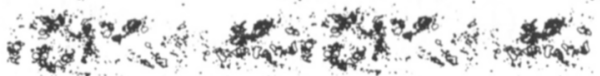

Fig. 9. Correlation of the large-scale distribution of solar magnetic fields with the interplanetary magnetic field sector structure drawn in Carrington's coordinates for Rotations Nos. 1546-1555. On the left side of the figure successively mounted magnetic synoptic charts of the negative polarity, on the right side of the positive polarity, distribution are shown. In the middle part of each figure the negative sector fields (to the left) and the positive sector fields (to the right) are drawn. 

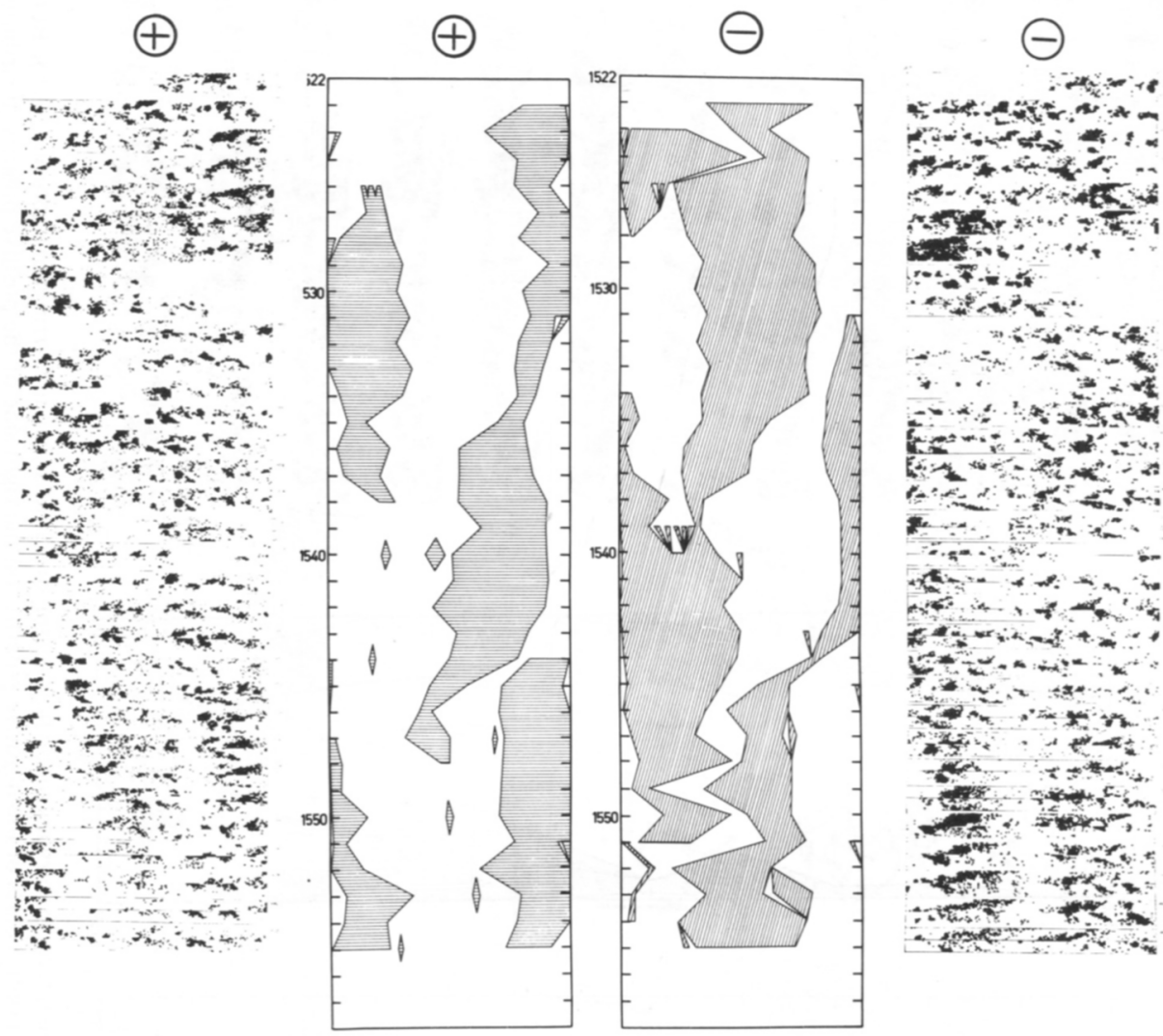

Fig. 10. Correlation of the secondary rows and streams (28-29 day synodic rotation) of solar magnetic fields from higher latitudinal zones $\left( \pm 20^{\circ}- \pm 40^{\circ}\right)$ with the interplanetary magnetic field sector structure is shown. The negative polarity fields are demonstrated to the right, the positive polarity fields to the left of the figure. The interplanetary fields are drawn in Carrington's coordinates. The shift of centers of gravity of individual interplanetary field sectors coinciding with the shift of 28-29 day successions of rows of the solar fields as well as practically no shift of sector's boundaries in Carrington's network may be seen.

part of our information concerns the morphology and only some findings deal with the kinematics of the field distribution. Although there exist some regularities and recurrences, without a theoretical model of the process leading to such a distribution it is difficult to understand it. We spoke about the role of some agents influencing that distribution, especially about the differential rotation, but we did not discuss the effect of other important influences such as for example the convection and motions in general, although it seems that motions, before all the convective motions may play a very important role.

It is very difficult to explain the observed distribution regularities using the known semi-empirical models of the magnetic field distribution changes depending on the phase of the solar activity cycle (Babcock, 1961; Leighton, 1964). Especially difficult to explain is the regular zonal as well as sectoral distribution of background fields, the different role of both polarities, such as, for example, the better visibility of 'supergiant' structures in the negative polarity fields, the close relation of these 
negative polarity fields to the young solar activity regions and to the 28-29-day rows or successions of sections and the better visibility of 'giant' granules in the positive polarity fields, their relation to the remainders of old activity which seem to be the source of the solar wind, and their better evidence of the 27-day rows in their distribution. The same is true about the mutual interaction of both types of rows and the development of huge regularities occupying half of the solar surface and connected with the interplanetary field sector structure.

The major part of recent attempts to construct a model of observed regularities in field and activity distribution uses the interplanetary magnetic field sector structure as a starting point. We may quote as an example the papers by Svalgaard et al. (1974), Antonucci (1974). But there are also theoretical papers trying to explain some of the observed behaviours of the large-scale distribution of solar magnetic fields (Stix, 1971; Yoshimura, 1973; Wolff, 1974). Let us hope that the present Symposium will stimulate an increased interest in these problems.

\section{Acknowledgements}

I wish to thank Dr R. Howard from the Hale Observatories, Carnegie Institution of Washington, California Institute of Technology, Pasadena, for supplying me with the synoptic charts of the photospheric magnetic fields constructed from the daily observations of the Mt. Wilson Observatory prior to their publication.

\section{References}

Altschuler, M. D., Newkirk, G. Jr., Trotter, D. E., and Howard, R.: 1971, in R. Howard (ed.), 'Solar Magnetic Fields', IAU Symp. 43, 588.

Altschuler, M. D., Trotter, D. E., Newkirk, G. Jr., and Howard, R.: 1974, Solar Phys. 39, 3.

Ambrož, P., Bumba, V., Howard, R., and Sýkora, J.: 1971, in R. Howard (ed.), 'Solar Magnetic Fields', IAU Symp. 43, 696.

Antonucci, E.: 1974a, Solar Phys. 34, 471.

Antonucci, E.: 1974b, SUIPR Report No. 570, Inst. for Plasma Research, Stanford Univ.

Antonucci, E. and Svalgaard, L.: 1974, Solar Phys. 36, 115.

Babcock, H. D.: 1959, Astrophys. J. 130, 364.

Babcock, H. W.: 1961, Astrophys. J. 133, 572.

Babcock, H. W. and Babcock, H. D.: 1955, Astrophys. J. 121, 349.

Bumba, V.: 1967, Hierarchy of Solar Magnetic Field Distribution, Moscow.

Bumba, V.: 1970a, in E. R. Dyer (ed.), Solar Terrestrial Physics, Part I, Dordrecht, p. 21.

Bumba, V.: 1970b, Solar Phys. 14, 80.

Bumba, V.: 1972a, in C. P. Sonett et al. (eds.), Solar Wind, Washington, p. 31.

Bumba, V.: 1972b, in C. P. Sonett et al. (eds.), Solar Wind, Washington, p. 151.

Bumba, V.: 1976a, Bull. Astron. Inst. Czech. 27 (in press).

Bumba, V.: 1976b, Bull. Astron. Inst. Czech. 27 (in press).

Bumba, V. and Howard, R.: 1956a, Astrophys. J. 141, 1492.

Bumba, V. and Howard, R.: 1965b, Astrophys. J. 141, 1502.

Bumba, V. and Howard, R.: 1969, Solar Phys. 7, 28.

Bumba, V. and Obridko, V. N.: 1969, Solar Phys. 6, 104.

Bumba, V. and Růžičková-Topolová, B.: 1969, Bull. Astron. Inst. Czech. 20, 63.

Bumba, V. and Sýkora, J.: 1973, in M. J. Rycroft and S. K. Runcorn (eds.), COSPAR Space Research, Vol. XIII, Berlin, p. 1973.

Bumba, V. and Sýkora, J.: 1974, in G. Newkirk, Jr. (ed.), 'Coronal Disturbances', IAU Symp. 57, 73. 
Bumba, V., Howard, R., Kopecký, M., and Kuklin, G. V.: 1969, Bull. Astron. Inst. Czech. $20,18$.

Bumba, V., Howard, R., Martres, M. J., and Soru-Iscovici, I.: 1968, in K. O. Kiepenheuer (ed.), 'Structure and Development of Solar Active Regions', IAU Symp. 35, 13.

Dittmer, P. H.: 1974, SUIPR Report No. 597, Prepr. Inst. for Plasma Research, Stanford Univ.

Howard, R.: 1974a, Solar Phys. 38, 283.

Howard, R.: 1974b, Solar Phys. 38, 59.

Howard, R.: 1974c, Solar Phys. 39, 275.

Howard, R. and Koomen, M. J.: 1974, Solar Phys. 37, 469.

Howard, R., Bumba, V., and Smith, S. F.: 1967, Carnegie Inst. of Washington Publ. No. 626, Washington.

Knoška, S.: 1976, Bull. Astron. Inst. Czech. 27 (in press).

Krüger, A., Bumba, V., Howard, R., and Kleczek, J.: 1968, Bull. Astron. Inst. Czech. 19, 180.

Leighton, R. B.: 1964, Astrophys. J. 140, 1547.

Martres, M. J., Pick, M., and Parks, G. K.: 1970, Solar Phys. 15, 48.

Ness, N. F. and Wilcox, J. M.: 1966, Astrophys. J. 143, 23.

Severny, A. B., Wilcox, J. M., Scherrer, P. H., and Colburn, D. S.: 1970, Solar Phys. $15,3$.

Schatten, K. H., Ness, N. F., and Wilcox, J. M.: 1968, Solar Phys. 5, 240.

Stenflo, J. O.: 1972, Solar Phys. 23, 307.

Stix, M.: 1971, Astron. Astrophys. 13, 203.

Svalgaard, L.: 1972, J. Geophys. Res. 77, 4027.

Svalgaard, L. and Wilcox, J. M.: 1974, SUIPR Report No. 605, Prepr. Inst. for Plasma Research, Stanford Univ.

Svalgaard, L., Wilcox, J. M., and Duvell, T. L.: 1974, SUIPR Report No. 537, Inst. for Plasma Research, Stanford Univ.

Sýkora, J.: 1971, Bull. Astron. Inst. Czech. 22, 12.

Sýkora, J.: 1973, Contr. Astron. Obs. Skalnaté Pleso 5, 5.

Švestka, Z.: 1968, Solar Phys. 4, 18.

Vitinskij, Yu. I.: 1969, Solar Phys. 7, 210.

Vitinskij, Yu. I.: 1973, Ciklichnost i prognosy solnechnoj aktivnosti, Naukâ, Leningrad.

Vitinskij, Yu. I.: 1974, Bull. Astron. Inst. Czech. 25, 222.

Wilcox, J. M.: 1968, Space Sci. Rev. 8, 258.

Wilcox, J. M.: 1972, Comments Astrophys. Space Phys. 4, 141.

Wilcox, J. M. and Howard, R.: 1968, Phys. Rev. Letters 20, 1252.

Wilcox, J. M. and Ness, N. F.: 1965, J. Geophys. Res. 70, 5793.

Wilcox, J. M. and Scherrer, P. H.: 1972, J. Geophys. Res. 77, 5385.

Wilcox, J. M. and Svalgaard, L.: 1974, Solar Phys. 34, 461.

Wilcox, J. M., Severny, A. B., and Colburn, D. S.: 1969, Nature 224, 353.

Wolff, Ch. L.: 1974, Astrophys. J. 194, 489.

Yoshimura, H.: 1973, Solar Phys. 33, 131.

\section{DISCUSSION}

Krause: I would like to come back to the point, where you speak about the behaviour of the Sun as a magnetic monopole. I reject the assumption that the magnetic field is not source-free. Then this behaviour of the magnetic field indicates electric currents in higher levels than the photosphere. My question is are there other indications of subphotospheric currents at times, when the magnetic field behaves like a 'magnetic monopole'?

Bumba: I do not know such observations that might answer your question. As far as I know such indications are not observed, although the 'monopole' behaviour of the solar magnetic field is observed relatively often.

Stix: The identification of interplanetary sector boundaries with photospheric sector boundaries is difficult for two reasons: Firstly, streams in the solar wind may influence the position of sector boundaries at $1 \mathrm{AU}$, and secondly, closed field configurations near the Sun cause large deviations from the simple spiral field pattern.

Bumba: Nevertheless any 'supergiant' regular structure that I could correlate with the interplanetary sector structure shows the dependence of the position of its sector boundary on the situation I mentioned in my talk.

Vandakurov: May we consider the extended magnetic field with the largest scale to be a superposition of a rigidly rotating simple sector structure and a differentially rotating sector structure? 
Bumba: As far as I know such a model of superimposed two kinds of magnetic field distribution has been suggested by Dr Wilcox. Maybe Dr Wilcox wishes to say a few words concerning this model himself.

Wilcox: There may exist a sector structure somewhere below the photosphere. These field lines have rigid rotation. When they rise to the photosphere (perhaps by magnetic buoyancy) the magnetic lines are distorted by differential rotation. Thus the observed photospheric field can display both rigid rotation (on a long time scale, i.e. several rotations) and differential rotation (on a shorter time scale of days). 